\title{
Tipo de cambio: Políticas cambiarias aplicadas en Costa Rica, 1960-2020
}

\section{Currency rate its evolution from 1960 to 2020 and the exchange policies applied in Costa Rica}

DOI 10.22458/rna.v12i2.3916

Mag. Rosa María Vindas-Chaves

Universidad Estatal a Distancia, Oficina de Recursos Humanos, San José, Costa Rica

rvindas@uned.ac.cr; https://orcid.org/0000-0002-7434-9016

\section{RESUMEN}

Las variaciones que presenta el tipo de cambio tienden a modificar el poder adquisitivo de los ciudadanos de un país, de manera que cuando el valor de la moneda extranjera se aprecia, las personas con el mismo ingreso bruto en moneda nacional tienen menos posibilidades de adquirir bienes $\mathrm{y}$ servicios. El presente artículo muestra el resultado de una investigación descriptiva y bibliográfica, es un estudio de un periodo aproximado de 60 años de los datos de tipo de cambio, de compra, venta o promedio, que brindan los insumos requeridos para comprender las implicaciones de los cambios históricos en Costa Rica y la respectiva política cambiaria.

\section{RÉSUMÉ :}

Les variations du taux de change ont tendance à modifier le pouvoir d'achat des citoyens d'un pays, de sorte que lorsque la valeur de la monnaie étrangère est appréciée, les personnes ayant le même revenu brut en monnaie nationale ont moins de possibilités d'acquérir des biens et des services. Cet article montre le résultat d'une recherche descriptive et bibliographique, est une étude sur une période d'environ 60 ans des données de taux de change, d'achat, de vente ou de moyenne, qui fournissent les apports nécessaires en ce qui concerne les implications des changements historiques au Costa Rica et la politique de change correspondante.

\begin{abstract}
:
The variations in the currency rate tend to modify the purchasing power of the citizens of a country, so that when the foreign currency revalues, people with the same gross income in national currency are less likely to acquire goods and services. This article shows the result of a descriptive and bibliographic research, it is a study of an approximate period of 60 years of the exchange rate, purchase, sale or average data, which provide the required inputs in relation to the implications of the historical changes in Costa Rica and the corresponding currency policy.
\end{abstract}

\section{RESUMO:}

As variações que apresenta a taxa de câmbio tendem a modificar o poder aquisitivo dos cidadãos de um país, de forma que quando o valor da moeda estrangeira se aprecia, as pessoas com a mesma renda bruta em moeda nacional têm menos possibilidades de adquirir bens e serviços. Este artigo apresenta o resultado de uma investigação descritiva e bibliográfica, trata-se de um estudo de um período aproximado de 60 anos dos dados da taxa de câmbio, compra, venda ou média, que fornecem os insumos necessários em relação às implicações das mudanças históricas na Costa Rica e a respectiva política cambial.
PALABRAS CLAVE: LIBERALIZACIÓN CAMBIARIA, BANDAS CAMBIARIAS, MINIDEVALUACIONES, APRECIACIÓN.
KEYWORDS:

CURRENCY LIBERALIZATION, CURRENCY BANDS, MINIDEVALUATIONS, REVALUATION.
MOTS-CLÉS :

LIBÉRALISATION DES TAUX DE CHANGE, FOURCHETTES DE TAUX DE CHANGE, MINIDÉVALUATIONS, APPRÉCIATION.
PALAVRAS-CHAVE: LIBERALIZAÇÃO DO CÂMBIO, BANDAS CAMBIAIS, MINI DESVALORIZAÇÕES, APRECIAÇÃO. 


\section{INTRODUCCIÓN}

Las decisiones en materia económica de un país dependen de una serie de variables que por lo general se ajustan de forma automática, de acuerdo con las fuerzas de oferta y demanda del mercado que definen los precios de los bienes y servicios, al igual que los precios de los factores de producción. El valor que asumen los mismos a lo largo de la historia está sujeto a lo que se conoce como la mano invisible del mercado. Esta representa indicadores económicos o datos de carácter estadístico, que permiten hacer análisis de la situación económica de un país, y adquieren especial importancia por las implicaciones que tienen sobre la economía en general, por ello son particularmente relevantes. Si bien son determinados por el mercado, en algunas oportunidades las autoridades deben intervenir para la fijación de precios, por el impacto que tienen en la economía como un todo.

Una de estas variables o indicadores económicos es el tipo de cambio, el cual afecta las decisiones de consumo de los habitantes de un país. Ante la imposibilidad de conseguir en un solo documento el total de datos de esta variable, tanto en compra y venta, como en un promedio a lo largo de su historia en Costa Rica y ante el aumento que el tipo de cambio muestra a inicios del año 2021, se realiza un estudio de la evolución que ha tenido el tipo de cambio del dólar americano o estadounidense en Costa Rica en los últimos sesenta años (para los efectos de este artículo se le denominará con el nombre de dólar).

Sobre el precio de este indicador, el Banco Central de Costa Rica (BCCR) tiene injerencia directa según la política monetaria que el gobierno en turno haya definido. En distintos momentos de su historia, la velocidad de su crecimiento ha impactado de forma directa el poder adquisitivo y el bienestar de los costarricenses. Sin embargo, este será un tema por demostrar en una investigación que correlacione este indicador con indicadores de bienestar económico.

Con relación a la conformación de este artículo, se presentará una recopilación de la información estadística de este indicador, desde el año 1960 hasta el año 2020. Se mostrará tanto el precio de compra, como el de venta a diciembre de cada uno de los años del último medio siglo, así como el promedio del precio del dólar mensual en los últimos sesenta años. Se evidencian, para los momentos de cambio más relevantes a lo largo de este periodo, los datos de modificación diaria del dólar al precio de compra y venta del mismo, pero a partir del año 1983 se presentará el dato diario. Se detallarán la fecha y el periodo requerido para aumentos de cada $₫ 50,00$, así como el valor que alrededor de los ф500,00 se presentó por más tiempo en el mercado. Además, se hará una breve referencia a las diferentes modalidades de política cambiaria que se han aplicado en Costa Rica en las últimas seis décadas.

Se podrá observar la afectación que tuvo este indicador en el cierre de la segunda década del siglo XXI, producto de la pandemia por COVID-19. Dicha pandemia incidió directamente en las relaciones comerciales y las fuerzas de oferta y demanda del dólar en todo el mundo, situación que también tiene su impacto en el tipo de cambio en Costa Rica. Sobre todo por la falta del ingreso de dólares, ya que por más de 8 meses del año 2020 se cerraron las fronteras aéreas, con lo que el ingreso de divisas por turismo se vio fuertemente afectado. Sin embargo, aun así la variación relativa no llega a las proporciones que se pueden visualizar en la década de los años ochenta del siglo XX en Costa Rica. Es decir, aún con las implicaciones de esta pandemia, el efecto en el tipo de cambio en el 2020 no ha sido el más fuerte de la historia, y eso se evidenciará con la serie de datos que se recopilan y se presentan en este documento (Schatan, 1998).

La información de la serie histórica a partir del año 1983 se encuentra disponible en el BCCR y es factible ubicar la variación diaria. La información cambiaria antes de esta fecha debe ser ubicada en otros documentos generados por el mismo BCCR, pero que deben ser solicitados de forma expresa. Un detalle importante que el BCCR deja claro en su página es que antes de noviembre de 1983 estos datos corresponden a un mercado intercambiario, de 1983 a marzo de 1992 corresponde a un mercado libre intercambiario, y de esa fecha en adelante estos datos se convierten en un tipo de cambio de referencia del BCCR. Es decir que el valor que presente el BCCR, al ser solo de referencia deja una franja de acción a las entidades bancarias para que establezcan el valor al que compran y venden en la ventanilla de su banco.

La evolución del tipo de cambio del dólar, en referencia a las variaciones relativas de este indicador, como muchas otras variables en la economía tiene un comportamiento de crecimiento relativo cíclico, lo cual será mostrado en la información de este artículo. Esto afecta directamente el valor que presentan otra serie de indicadores de un país, por ejemplo, la inflación, el producto interno bruto (PIB), el crecimiento económico y el bienestar económico. Por ello, es importante poder visualizar otros momentos de depreciación o devaluación significativa, para poder estudiar los efectos en la economía y cómo se podrían prevenir afectaciones que en el pasado se relacionan directamente como productos de fuertes aumentos en el tipo de cambio del dólar. 
A partir de las series históricas que se recopilan en este artículo y que se presentan en forma consolidada, al mostrar la evolución del tipo de cambio del dólar en Costa Rica, pueden surgir investigaciones sobre análisis comparativos con otros indicadores. Además, esta investigación puede funcionar como base o referencia para efectos académicos.

\section{METODOLOGÍA}

Este trabajo investigativo se realiza bajo un enfoque que combina dos métodos de investigación: el método descriptivo, ya que se centra en la ubicación de los datos como objeto de estudio, en este caso el tipo de cambio a lo largo de la historia del periodo definido, y el método bibliográfico, debido a que se proceden a explicar las políticas cambiarias aplicadas, que se asocian con la trayectoria de los datos. La justificación de la escogencia del enfoque se fundamenta en el hecho de que este se ajusta a las necesidades y condiciones propias del entorno y ambiente que rodean al objeto de este estudio.

Lo anterior se acompaña con un análisis descriptivo y una revisión bibliográfica que incluye a los autores más citados y reconocidos en el tema. Además, se complementa con el criterio experto de la autora, en el seguimiento de este tema en los últimos veinte años, tanto en su condición de consultora como de docente.

\section{COYUNTURAS HISTÓRICAS Y EL TIPO DE CAMBIO}

En la segunda década del siglo XXI, en Costa Rica y el mundo entero se vivió una de las crisis económicas más fuertes de la historia cercana, la cual se complica aún más por una pandemia sin precedentes que nace en Wuhan, China, a finales del año 2019. Esta será recordada como la pandemia por COVID-19, que quedará marcada en la historia mundial como un punto de referencia, pues detuvo la economía y comercio mundiales.

La historia de la colaboración económica y de la incorporación de Costa Rica a organismos internacionales como el Fondo Monetario Internacional (FMI), "organismo intergubernamental creado en 1945 por la ONU en base a los acuerdos de Bretton Woods cuyos objetivos son promover políticas cambiarias sostenibles a nivel internacional, facilitar el comercio internacional y reducir la pobreza a nivel mundial" (Blokhin, 2021, párr. 4), vuelve a tomar protagonismo en las decisiones trascendentales del país, como se tuvo en el momento de su creación, y que marcó el tipo de cambio fijo que, desde los años cincuenta hasta los setenta, se tuvo en Costa Rica.

Para mediados del siglo XX la economía costarricense se regía por un modelo económico de sustitución de importaciones. Esto comenzó a vislumbrarse desde finales de los años cincuenta, y desembocó a principios de la década de los sesenta en la promoción de un amplio proceso de industrialización y de integración económica. Fue en esos años que se inició en Costa Rica la implementación del llamado Modelo de Sustitución de Importaciones (MSI). Según Villasuso (2000), es un:

Modelo impulsado por la CEPAL en América Latina, y el cual se puso muy de moda entre los países subdesarrollados. Este sistema consistía en otorgarle incentivos a la industria local, con el propósito de que orientara su producción hacia el mercado interno, sobre todo de los bienes que normalmente se importaban. (p. 6)

Es en esta época donde nacen instituciones como la Comisión Económica para América Latina y el Caribe (CEPAL), que para el 2021 en Costa Rica vuelve a tener un papel preponderante, para lograr la aprobación de créditos que permitan solventar la situación económica del país (Saborío-Muñoz, 1997).

En la época de los años cincuenta del siglo pasado, uno de los acuerdos de Bretton Woods, ciudad donde nace el FMI, fue que el tipo de cambio de Costa Rica se establecía básicamente como un tipo de cambio fijo y así se cumple hasta los años setenta. A pesar de este deterioro en el comercio regional, durante la década de los setenta Costa Rica tuvo acceso a una gran cantidad de recursos financieros, producto de los excedentes generados por la producción de petróleo a los países productores, y al aumento vertiginoso en los precios internacionales del café, el cual era su principal producto de exportación. Esta afluencia de recursos externos permitió ignorar durante unos años los problemas que existían en el sistema productivo costarricense. Cabe recalcar que durante todo el periodo el tipo de cambio permaneció prácticamente fijo, excepto por algunas pequeñas devaluaciones, lo cual iba muy en línea con lo estipulado por el acuerdo de Bretton Woods (Wonnacott y Wonnacott, 1981; Saborío-Muñoz, 1997).

Medio siglo después y al pasar por diferentes políticas cambiarias, la participación del FMI sigue presente en las políticas que debe tomar Costa Rica. El problema cambiario empieza a evidenciar su impacto en diferentes 
indicadores económicos, producto de los niveles de endeudamiento excesivo y la poca liquidez que no le permite al Gobierno poder maniobrar, pues el mayor porcentaje del PIB se debe dedicar al pago de la deuda pública y lo que resta debe dedicarse al pago de la planilla estatal (Chinchilla, 2014).

Este panorama recuerda lo pasado en los años ochenta, aunque por motivos diferentes, pues en dicho periodo el problema enfrentado fue producto de un aumento en las tasas internacionales de interés, lo cual hizo que el presidente Rodrigo Carazo Odio de los años 1980 a 1982 declarara moratoria de la deuda en Costa Rica.

La crisis fue entonces inevitable. Las reservas monetarias internacionales se agotaron rápidamente y, a mediados de 1981, Costa Rica se vio obligada a comunicar a los bancos acreedores la imposibilidad de pagar el principal y los intereses de su deuda externa. (Lizano y López, 2006, p. 226)

En materia cambiaria en Costa Rica, se pasa en 1979 de un tipo de cambio que venía fijo desde 1970 en $₫ 8,60$ hasta diciembre de 1979, a ф14,31 por dólar a diciembre del 1980, y para finales del año 1981 se cierra con $\notin 36,18$ como tipo de cambio de venta del dólar. Esta situación, como se evidenciará, hace que en menos de una década de 1979 a 1981, el tipo de cambio en términos relativos se devalúe en un $881 \%$, cambio relativo que aún en los dos primeros meses del año 2021 no se ha vuelto a presentar.

No obstante, la historia reciente, específicamente desde finales del año 2018, evidencia que la economía costarricense vuelve a presentar una serie de problemas con los que el panorama económico se complica. En este caso, dentro de los factores que son la base de esta situación apremiante se pueden mencionar el proceso electoral (donde el pluripartidismo se consolida) y el déficit fiscal que se complica a partir del año 2006, año en que ingresos fiscales y gastos fiscales lograron un equilibrio por última vez.

De acuerdo con las publicaciones de la época, el problema del tipo de cambio obedecía a que, según el presidente del Banco de Costa Rica:

Existían varios factores que estaban empujando el tipo de cambio al alza. Entre ellos, el aumento de la tasa de interés de los Estados Unidos, el aumento en el precio del petróleo, así como la situación de incertidumbre en el país por el rumbo de las finanzas públicas. (Delfino, 2018, párr. 8)

En el problema de las finanzas públicas sobresalían la discusión de las altas pensiones y la estructura salarial del empleo público. Temas que afectan directamente el PIB vía gastos y hace prever problemas económicos, los cuales se complican por las limitaciones para conseguir el financiamiento, que permita hacer frente a las deudas pactadas con altos intereses y de los gastos desmesurados del sector público.

El déficit fiscal y la necesidad de concertar una forma de generar ingresos tributarios y controlar el gasto público, llevaron a que en los meses de octubre y noviembre de 2018 se generaran una serie de rumores y la imposibilidad de colocar bonos del Gobierno, y otros mecanismos para recolección de liquidez para enfrentar los gastos. Esto provocó señales de riesgo que evidentemente han iniciado la afectación en una serie de variables económicas reales que han despertado el interés en su estudio. ${ }^{1}$

Una de las variables que a corto plazo más afectó de forma inmediata y directa en ese momento histórico a la población fue el tipo de cambio, el cual generó una moratoria significativa en los créditos de vivienda y automóviles en el sistema bancario nacional para el 2018. Lo anterior, producto del aumento de casi $\not 10,00$ que se hace el 7 de noviembre de 2018, fecha para la cual el tipo de cambio de venta del dólar alcanza su máximo valor absoluto de ф631,30.

La política monetaria de mayor flexibilidad cambiaria que el BCCR decidió establecer en materia del tipo de cambio del dólar desde el mes de agosto de 2018, fue anunciada por don Rodrigo Cubero su presidente, lo cual generó en esta época un aumento del tipo de cambio (de $\mathbb{\$} 575$ hasta $\mathbb{\$} 631$ ) que ronda el $10 \%$ de incremento en un periodo que abarca los meses de agosto a noviembre de 2018. Esto se evidencia en el periódico La Nación del 23 de agosto de 2018, en el apartado de política económica (Leitón y Ramírez, 2018).

1 El tema del hoyo fiscal, y los problemas que desde el 2017 enfrenta Costar Rica se detalla en: https://www.larepublica.net/noticia/ hueco-fiscal-actuacion--del-gobierno-solis-o-engano-en-caprudentempana-electoral 
Las afectaciones de esta devaluación impactaron sobre todo en los costarricenses asalariados, que al tener ingresos en colones habían acudido al sistema financiero y se endeudaron en dólares, de modo que en poco tiempo sus pasivos se elevaron en la moneda local y sus gastos mensuales también se incrementaron por el aumento del pago de las cuotas de dichas deudas. Sin embargo, la contraparte que son los ingresos, se mantuvieron sin variación alguna, sobre todo porque de conformidad con la política monetaria del BCCR, se apostaba por mantener el índice de inflación en niveles que no superaban el $3 \%$, por lo que difícilmente se podían esperar aumentos salariales producto de ajustes al costo de vida.

Por todas estas razones, para entender mejor este tema y poder visualizar la evolución del tipo de cambio del dólar se presentan los datos consolidados de las últimas seis décadas en un solo cuadro. Al contar con la evolución de los datos y las políticas que se han implementado para determinar el mismo, la autora del presente artículo considera que serán de mucha ayuda a los tomadores de decisiones, o para los investigadores y estudiantes que requieran de estos datos consolidados, con el fin de generar relaciones econométricas, y buscar las relaciones de la variación del tipo de cambio en referencia a otros indicadores de interés.

Para una mejor compresión del tema se presentará una breve explicación teórica del tipo de cambio y los principales términos del mismo. Al tener claro estos aspectos, se elabora un resumen mensual del tipo de cambio respecto al dólar, que abarca desde 1970 hasta el mes de diciembre de 2020, tanto en referencia al precio de compra como al de venta, así como un promedio mensual del precio de venta, para que se pueda visualizar su evolución en estos sesenta años. Se indicará y explicará la política cambiaria bajo la cual se ha manejado en Costa Rica el tipo de cambio para cada época.

\section{EL TIPO DE CAMBIO}

El tipo de cambio nominal, según Mankiw (2009), es "la tasa a la que una persona puede intercambiar la divisa de un país por la de otro país" (p. 703). Esta representa la cantidad de unidades de una moneda que hay que entregar para obtener una unidad de moneda extranjera (Heilbroner y Lester, 1987; Samuelson y Nordhaus, 1992; Parkin, 1995; Krugman y Obstfeld, 2006).

Al igual que cualquier otro bien de la economía, el precio del tipo de cambio se establece por las fuerzas de mercado, es decir, por la interacción de la oferta y demanda de divisas prevalecientes en un momento dado. Esta representa el punto de equilibrio entre la cantidad de moneda extranjera que se requiere para hacer las transacciones de bienes y servicios en esa moneda, contra lo que los diferentes agentes económicos del mercado ofrecen de la misma (dentro de estos agentes el BCCR juega un papel muy importante en la estabilización de la divisa estadounidense). "Ese lugar donde se determina el precio de la moneda extranjera se conoce como "Mercado de divisas Extranjeras" o "Mercado de Cambios Exteriores" y funciona como cualquier otro" (Tacsan-Chen, 2001, p. 100).

Cuando el tipo de cambio sube o baja, estas variaciones pueden ser producto de:

1) Las fuerzas de oferta o demanda de las divisas asociadas con políticas cambiarias flexibles o flotantes.

2) Por decisiones de política económica de las autoridades monetarias.

Si el tipo de cambio sube se conoce con el nombre de devaluación, y si baja se le llama revaluación. Por lo general, estas se asocian con la confianza en la economía de un país, es decir, son los cambios en la divisa producto de la inestabilidad o estabilidad de la economía del país, que a su vez se relaciona con las políticas económicas del gobierno en turno.

El tipo de cambio real, según Salvatore (1994), “corresponde a la tasa nominal dividida entre el índice de precios al consumidor" (p. 683), lo que refleja la tasa a la que una persona puede intercambiar bienes y servicios de un país por bienes y servicios de otro país. Esta es la variable o índice relevante en la economía, en lo referente a la producción nacional, pues es el que evidencia si los productos de un país son más baratos o más caros que los del resto del mundo. Por fórmula se obtiene de la siguiente manera: 


\section{$\operatorname{Tcr}=\operatorname{Tcn} * \underline{\mathrm{IPC} r}$}

\section{IPCcr}

Tcr $=$ Tipo de cambio real.

Tcn $=$ Tipo de cambio nominal.

IPCrm = Índice de precios del resto del mundo.

IPCcr = Índice de precios de Costa Rica.

Como se puede apreciar en la fórmula, el tipo de cambio real está afectado directamente por la inflación del resto del mundo, pues si en los otros países la inflación aumenta el tipo de cambio real también aumenta. Por ello la producción se hace más competitiva, pero tiene una relación inversa con la inflación interna, de manera que si la inflación aumenta, la producción nacional se vuelve menos competitiva con las consecuencias que esto puede traer al resultado final del PIB del país.

El que el tipo de cambio se aprecie o se revalúe, quiere decir que se deben dar menos monedas de un país por la divisa del otro país; esto pocas veces se ha presentado en Costa Rica. En la década del 2000 es cuando este fenómeno más se presenta, dos eventos específicos se pueden ubicar si se compara el precio de venta del dólar de diciembre de 2006 a diciembre de 2007, donde la moneda, según el precio de referencia para venta del BCCR, se revalúa al pasar de $\mathbb{\Downarrow} 519,95$ a $\llbracket 500,97$ por dólar, de modo que se da un 3,65 \% de disminución en el tipo de cambio. El caso de mayor revaluación se encuentra en los mismos términos de referencia, pero del 2009 al 2010, donde la divisa se aprecia al

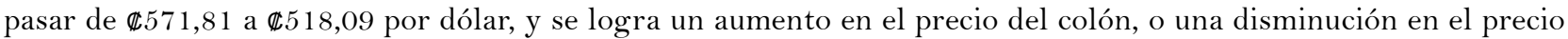
del dólar de un 9,39\%.

A lo largo de la historia de Costa Rica las devaluaciones son más frecuentes, producto de las fuerzas de mercado que es cuando la divisa extranjera, en este caso el dólar, aumenta de valor con respecto al colón. La devaluación relativa más alta de la historia de Costa Rica se ubica entre 1980 y 1981, en los que de diciembre a diciembre el tipo de venta del dólar pasó de $\mathbb{1} 14,31$ a $\llbracket 36$ por cada dólar, y se registró un aumento del 152,83\%. En términos absolutos, la devaluación más grande, por incertidumbre de mercado, corresponde al tipo de cambio de referencia del BCCR que se reportó el 7 de noviembre de 2018, con un valor de $\mathbb{6} 631$,30 por dólar.

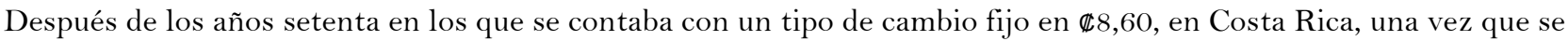
dejó su definición a las fuerzas del mercado y con intervención del Gobierno, se ubica entre el mes de febrero de 2006

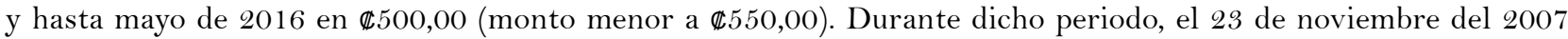

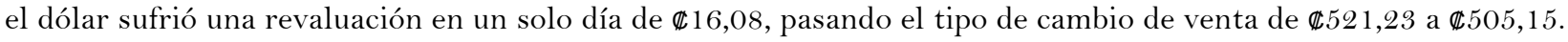

\section{DETERMINANTES DEL TIPO DE CAMBIO REAL}

El tipo de cambio real de equilibrio no es un número inmutable, por el contrario, cada vez que algunas de las variables reales fundamentales sufren una variación, también deberá cambiar el tipo de cambio de equilibrio. Cualquier desviación del tipo de cambio real con respecto a uno del año base representa una situación de desequilibrio.

Teóricamente, muchos autores pueden enumerar varios factores para estas situaciones, los enfoques modernos mencionan los siguientes:

1) Términos internacionales de intercambio.

2) Flujos externos de capital que influyen en transferencias y donaciones.

3) El sistema de restricciones al comercio internacional que existe en el país.

4) El sistema tributario del país.

5) Tasa internacional de interés.

6) Composición del gasto del Gobierno. 
7) El ritmo de progreso tecnológico existente en el país.

8) Gustos y preferencias de consumidores.

9) La política cambiaria que defina el Gobierno.

Una variación en cualquier factor implica una modificación en relación con los precios comerciables internacionalmente con respecto a los no comerciables. Sin embargo, el análisis de las causas para las variaciones del tipo de cambio que se llegaron a presentar en cada una de las seis décadas de interés, es un tema que quedará pendiente.

\section{POLÍTICA CAMBIARIA}

Dentro de la economía existen una serie de variables que afectan la demanda agregada y la producción. Estas variables, por lo general, se dividen en dos grandes grupos:

1) Las que se denominan variables de política fiscal, pues afectan directamente la demanda agregada por medio del consumo (privado) o del gasto del Gobierno, donde se maneja las variables: gasto del Gobierno y los impuestos.

2) Por otra parte, se encuentra la política monetaria, en la cual, mediante el manejo de variables monetarias como las tasas de interés o el tipo de cambio, se logran movimientos en el mercado de dinero que afectan la tasa de interés o el tipo de cambio para variar la demanda agregada, ya sea por medio del gasto en inversión o de movimientos mediante el gasto en exportaciones netas, es decir, la diferencia neta entre exportaciones e importaciones.

Dentro de la política monetaria que es manejada en Costa Rica por el BCCR, existe la política cambiaria como una de las formas para movilizar la demanda agregada por medio de las exportaciones netas, y comprende el conjunto de acciones que toma el BCCR para lograr que el tipo de cambio se ajuste de forma real a las fuerzas de mercado cambiario. A lo largo de la historia cambiaria de Costa Rica se pueden distinguir diversas políticas cambiarias, a continuación se presenta un resumen de estas.

\section{POLÍTICA CAMBIARIA Y REGÍMENES CAMBIARIOS EN COSTA RICA 1960-2020}

Durante las últimas décadas, Costa Rica ha observado diferentes regímenes cambiarios. La corta historia cambiaria de Costa Rica puede dividirse en 8 etapas:

1) Antes del año 1957, Jorge Guardia (Chinchilla, 2014) detalla que para el año 1945 el tipo de cambio del

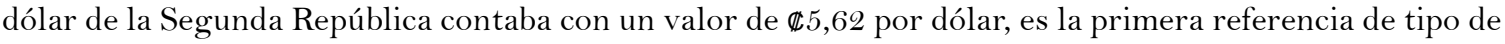
cambio que se puede ubicar en el BCCR.

2) De 1957 a 1960, el cambio nominal y real se mantuvieron sin variación gracias a la estabilidad internacional de las diferentes monedas y a que la inflación doméstica fue muy similar a la externa. A mediados de 1960 el tipo de cambio sufre una importante variación, lo cual culminó con una devaluación del colón de $\mathbb{E} 6,15$ a $₫ 6,65$ por dólar a la venta.

3) De 1962 a mediados de 1970 el tipo de cambio permaneció invariable, o sea se establece como un tipo de cambio fijo. En 1971 se recurrió a una devaluación discreta que llevó el precio del dólar a $\mathbb{4} 8,57$ a la compra y a $\mathbb{4} 8,60$ por dólar a la venta. Esta estabilidad respondía a los pactos del FMI y a los acuerdos de Bretton Woods.

4) De 1974 hasta 1979 el tipo de cambio nominal fue de $\mathbb{\&} 8,54$ la compra y $\mathbb{E} 8,60$ por dólar la venta. Este cambio se mantuvo temporalmente gracias a la bonanza cafetalera mundial y a la abundante disponibilidad de fondos externos. Sin embargo, una vez que los precios del café volvieron a los niveles normales y las divisas se redujeron, sobrevino la crisis cambiaria. Para ese entonces gobernaba el presidente Rodrigo Carazo Odio. En esta época, en Costa Rica existía el mercado negro del dólar y era común ubicar a intermediarios en lo que se conocía como los bajos del radio Monumental.

5) Para 1982, con el gobierno de Luis Alberto Monge, se adoptó el sistema cambiario de minidevaluaciones que rigió desde finales de 1983 hasta principios del 2005, a pesar de que en 1992 con la liberalización cambiaria se pretendió modificar la política cambiaria, pero al final las minidevaluaciones se mantuvieron. 
En la primera década de esta política es donde el tipo de cambio a precio de venta llega a tener una devaluación, cuya tasa de crecimiento supera el $800 \%$ producto de la crisis vivida a finales de los setenta. A partir de noviembre de 1983, el BCCR establece que el tipo de cambio corresponde al resultado de un mercado libre intercambiario. Es decir, el tipo de cambio establecido por esta entidad es solo un tipo de cambio de referencia, a diferencia de lo sucedido antes del año 1983, donde el tipo de cambio establecido era de igual aplicación en todo el sistema bancario nacional. De este momento en adelante, cada banco seguirá el valor dictado por el BCCR, pero podrán cobrar una diferencia en el precio, tanto de compra como de venta.

A pesar de la depreciación sufrida, se debe rescatar que, en este periodo, el sector exportador incrementó su renta, lo cual contribuyó al aumento y diversificación de esta importante actividad. Debido a que los ajustes fueron pequeños, los agentes contaron con bastante certeza para realizar sus planes de ahorro e inversión. No obstante, la implementación de este sistema generó algunos inconvenientes; en primer lugar, su aplicación automática y discrecional estuvo asociada con una tasa de inflación más alta y más variable. La inflación inercial surgió debido a que los distintos agentes económicos, incluyendo empresarios, financistas y los trabajadores, incorporaban anticipadamente el impacto de la devaluación en precios, tasas de interés y salarios.

Aparte de los inconvenientes anteriores propios del sistema de minidevaluaciones, es importante señalar que en términos generales esta política se diseñó de tal forma que los ajustes se realizaron sobre una base absoluta.

6) Para 1992, en el gobierno de Rafael Ángel Calderón Fournier y bajo la presidencia de Jorge Guardia en el BCCR, se asume una política de liberalización del tipo de cambio, se abre la cuenta de capitales de la balanza de pagos desde principios de 1992, y se decreta la flotación del colón. Durante el primer año se dan importantes entradas de capital privado, lo que contribuyó a que en 1992 se observara una estabilidad del tipo de cambio, donde solo se presenta una variación que no llega al $1 \%$, situación totalmente diferente a lo sucedido en la década que precede esta política cambiaria. Pero, como lo indican Lizano y López (2006):

Este intento de flexibilizar el sistema cambiario tuvo corta vida. Pocos meses después de puesto en práctica, el BCCR volvió a tomar las riendas del mercado cambiario, para evitar la continua apreciación nominal del colón que ocurrió durante la vigencia de la flotación cambiaria. Tal como se menciona en Delgado (2000, p. 446) en referencia a las manifestaciones incluidas en un estudio no publicado de Miguel Loría para la Academia de Centroamérica. (2006, p. 102)

Por lo que se considera que para finales de 1992 y hasta el 2006, la política cambiaria retornó en la práctica a un sistema de minidevaluaciones, solo que con un sistema cambiario abierto en las cuentas de capitales.

En vista de los inconvenientes, las autoridades económicas propiciaron la adopción de un régimen de mayor libertad cambiaria. Este sistema se vio facilitado por la prevalencia de una serie de condiciones internas y externas muy favorables:

a) El país había logrado un adecuado manejo de la política fiscal y monetaria.

b) El tipo de cambio real había experimentado una importante depreciación.

c) La actividad turística crecía aceleradamente, lo cual era una fuente de divisas.

d) Los déficits se habían reducido considerablemente.

e) El BCCR experimentó una acumulación de reservas internacionales.

f) Las tasas externas de interés experimentaron una significativa disminución.

g) La recesión de algunas economías industrializadas y las políticas de reformas propiciaron una importante entrada de capitales privados.

Durante los meses de mayo a junio de 1992 el tipo de cambio experimentó reiteradas revaluaciones (apreciaciones), como consecuencia de un importante flujo de capital privado y de una menor intervención del BCCR en el mercado cambiario, por lo que pasa de un tipo de cambio de venta de $\mathbb{\$} 138,50$ el 1 de marzo, a $\mathbb{1} 129,41$ el 24 de junio de 1992.

1) El 17 de octubre del año 2006, con el presidente Dr. Oscar Arias Sánchez y bajo la administración en el BCCR del economista Francisco de Paula Gutiérrez, después de más de 22 años de una política de minidevaluaciones, se cambia la política cambiaria a una de bandas cambiarias. En ella se establecía un 
valor máximo de compra y venta del dólar y un valor piso, valores dentro de los cuales los diferentes bancos del país podían definir el tipo de cambio. Este sistema cambiario se manejó por más de 8 años, periodo donde la banda cambiaria llegó a contar con valores entre $\mathbb{\$} 866$ como techo y $\llbracket 500$ como piso (Delgado-Montoya, 2015, párr. 6).

2) En enero de 2015 el BCCR toma la decisión de pasar de un régimen de bandas cambiarias al régimen que rige hasta la actualidad, que es un sistema de flotación administrada. Este periodo inicia con un tipo de cambio de $\$ 545,53$ la venta, y el 7 de noviembre de 2018 llegó a presentarse el valor más alto en la historia del precio del dólar, $\mathbb{E} 631$ 1,30 hasta cerrar en diciembre de 2020 con un tipo de cambio de referencia de $\mathbb{4} 617,30$ la venta, que para los primeros meses de enero y febrero de 2021 se mantiene en el mercado.

\section{EVOLUCIÓN DEL TIPO DE CAMBIO}

En este proceso de evolución se presentará la historia, en números, del tipo de cambio. Todos los datos son obtenidos del BCCR. Para los primeros años de la historia cambiaria de Costa Rica, ya que se contaba con un mercado oficial y uno libre, estos se proporcionarán en series para los dos mercados. Del año 1970 a 1982 se expondrá el tipo de cambio solo del mercado intercambiario con las observaciones del tipo de cambio que se aplicaba. Además, se presentarán las principales variaciones de 1970 a 1982 por semana.

Tabla 1. Tipo de cambio mercado oficial de Costa Rica. Precio de compra, precio de venta y tasa de crecimiento en el periodo 1945-1982

\begin{tabular}{|l|l|l|}
\hline \multicolumn{1}{|c|}{ FECHA } & \multicolumn{1}{|c|}{ COMPRA } & \multicolumn{1}{c|}{ VENTA } \\
\hline De 1945 a 1946 & 5,61 & 5,62 \\
\hline De 1947 al 31-08-61 & 5,60 & 5,67 \\
\hline Del 01-09-61 al 19-03-73 & 6,62 & 6,65 \\
\hline Del 20-03-73 al 23-04-74 & 6,62 & 6,68 \\
\hline Del 24-04-74 al 09-12-81 & 8,54 & 8,60 \\
\hline Del 10-12-81 al 10-06-82 & 20,00 & 20,16 \\
\hline Del 11-06-82 al 17-09-82 & 20,00 & 20,25 \\
\hline 18-09-82 & 20,00 & 20,30 \\
\hline
\end{tabular}

Fuente: BCCR. El artículo 20 de la Ley 6999 del 16 de setiembre de 1985, prohíbe al BCCR operar en este mercado. Solamente se hace la excepción para que lo haga con los estudiantes que estaban registrados en el BCCR hasta el 10 de diciembre de 1981, según lo dispuesto en las normas 50 y 51 del Presupuesto Extraordinario de la República, publicadas en el Alcance n.o 22 de La Gaceta n.o 247 del 26 de diciembre de 1985. 
Tabla 2. Tipo de cambio mercado oficial de Costa Rica. Precio de compra, precio de venta y tasa de crecimiento en el periodo 1966-1979

\begin{tabular}{|c|c|c|}
\hline FECHA & COMPRA & VENTA \\
\hline De 1953 al 31-12-66 & 6,62 & 6,65 \\
\hline Del 25-04-67 al 22-10-67 & 7,00 & 7,03 \\
\hline Del 23-10-67 al 01-1 1-67 & 7,55 & 7,58 \\
\hline Del 02-1 1-67 al 18-02-68 & 7,77 & 7,80 \\
\hline Del 19-02-68 al 03-03-68 & 8,07 & 8,10 \\
\hline Del 04-03-68 al 18-04-68 & 8,22 & 8,25 \\
\hline Del 19-04-68 al 24-04-68 & 8,12 & 8,15 \\
\hline Del 25-04-68 al 30-04-68 & 8,07 & 8,10 \\
\hline Del 02-05-68 al 04-05-68 & 7,97 & 8,00 \\
\hline Del 06-05-68 al 13-05-68 & 7,92 & 7,95 \\
\hline Del 14-05-68 al 30-05-68 & 7,87 & 7,90 \\
\hline Del 31-05-68 al 25-06-68 & 7,82 & 7,85 \\
\hline Del 26-05-68 al 11-07-68 & 7,77 & 7,80 \\
\hline Del 12-07-68 al 19-07-68 & 7,72 & 7,75 \\
\hline Del 20-07-68 al 30-07-68 & 7,67 & 7,70 \\
\hline Del 31-07-68 al 15-08-68 & 7,57 & 7,60 \\
\hline Del 18-08-68 al 25-08-68 & 7,52 & 7,55 \\
\hline Del 26-08-68 al 10-09-68 & 7,42 & 7,45 \\
\hline Del 1 1-09-68 al 29-09-68 & 7,37 & 7,40 \\
\hline Del 30-09-68 al 28-01-69 & 7,32 & 7,35 \\
\hline Del 29-01-69 al 11-02-69 & 7,27 & 7,30 \\
\hline Del 12-02-69 al 17-04-69 & 7,07 & 7,10 \\
\hline Del 18-04-69 al 14-12-69 & 6,92 & 6,95 \\
\hline Del 15-12-69 al 23-12-69 & 6,62 & 6,95 \\
\hline Del 24-12-69 al 19-06-71 & 6,62 & 6,65 \\
\hline Del 23-06-71 al 01-07-71 & 7,50 & 7,53 \\
\hline Del 02-07-71 al 12-07-71 & 7,50 & 7,53 \\
\hline Del 16-07-71 al 18-08-71 & 8,57 & 8,60 \\
\hline Del 19-08-71 al 18-09-71 & 8,57 & 8,60 \\
\hline Del 20-09-71 al 1 1-10-71 & 7,87 & 7,90 \\
\hline Del 11-10-71 al 19-10-71 & 8,22 & 8,25 \\
\hline Del 20-10-71 al 19-03-73 & 8,57 & 8,60 \\
\hline Del 20-03-73 a 1979 & 8,54 & 8,60 \\
\hline
\end{tabular}




\begin{tabular}{|c|c|c|c|}
\hline REFERENCIA & $\mathrm{FECHA}$ & COMPRA & VENTA \\
\hline & $09-10-80$ & 12,20 & 12,30 \\
\hline & $13-10-80$ & 11,56 & 11,62 \\
\hline & $14-10-80$ & 12,00 & 12,06 \\
\hline & $15-10-80$ & 11,84 & 11,90 \\
\hline & $16-10-80$ & 11,74 & 11,80 \\
\hline & Del $17-10-80$ al $20-10-80$ & 11,54 & 11,60 \\
\hline & $21-10-80$ & 11,44 & 11,50 \\
\hline & $22-10-80$ & 11,29 & 11,44 \\
\hline & $23-10-80$ & 11,34 & 11,40 \\
\hline & Del $24-10-80$ al $27-10-80$ & 11,30 & 11,36 \\
\hline & $28-10-80$ & 11,20 & 11,26 \\
\hline & Del $29-10-80$ al 31-10-80 & 11,18 & 11,24 \\
\hline & $03-11-80$ & 11,20 & 11,26 \\
\hline & $04-11-80$ & 11,24 & 11,30 \\
\hline & $05-11-80$ & 11,20 & 11,26 \\
\hline & $06-11-80$ & 11,21 & 11,27 \\
\hline & $07-11-80$ & 11,27 & 11,33 \\
\hline & $10-11-80$ & 11,44 & 11,50 \\
\hline & $11-11-80$ & 11,60 & 11,66 \\
\hline & $12-11-80$ & 12,00 & 12,06 \\
\hline & $13-11-80$ & 11,96 & 12,02 \\
\hline & $14-11-80$ & 11,92 & 11,98 \\
\hline & $17-11-80$ & 11,87 & 11,93 \\
\hline & Del $18-11-80$ al 19-11-80 & 11,92 & 11,98 \\
\hline & $20-11-80$ & 11,97 & 12,03 \\
\hline & $21-11-80$ & 11,98 & 12,04 \\
\hline & 24-1 1-80 & 12,00 & 12,06 \\
\hline & $25-11-80$ & 12,09 & 12,15 \\
\hline & $26-11-80$ & 12,64 & 12,70 \\
\hline & $27-11-80$ & 13,09 & 13,15 \\
\hline & $28-11-80$ & 13,30 & 13,36 \\
\hline & $01-12-80$ & 13,94 & 14,00 \\
\hline & $02-12-80$ & 15,64 & 15,70 \\
\hline & $03-12-80$ & 14,50 & 14,56 \\
\hline En la tarde & Del 03-12-80 al 09-12-80 & 13,50 & 13,56 \\
\hline En la tarde & $10-12-80$ & 13,65 & 13,71 \\
\hline En la tarde & Del $10-12-80$ al $11-12-80$ & 13,75 & 13,81 \\
\hline En la tarde & Del $11-12-80$ al $12-12-80$ & 14,00 & 14,06 \\
\hline En la tarde & Del $12-12-80$ al $15-12-80$ & 14,25 & 14,31 \\
\hline En la tarde & Del $15-12-80$ al 16-1 1-80 & 14,69 & 14,75 \\
\hline En la tarde & Del 16-12-80 al 17-12-80 & 14,75 & 14,81 \\
\hline
\end{tabular}


Tipo de cambio: Políticas cambiarias aplicadas en Costa Rica, 1960-2020

\begin{tabular}{|c|c|c|c|}
\hline \multirow[t]{7}{*}{ En la tarde } & Del $18-12-80$ al 23-12-80 & 14,50 & 14,56 \\
\hline & Del 23-12-80 al 26-12-80 & 14,40 & 14,46 \\
\hline & Del 26-12-80 al 06-01-81 & 14,25 & 14,31 \\
\hline & $07-01-81$ & 14,00 & 14,06 \\
\hline & 08-01-81 & 13,95 & 14,01 \\
\hline & $09-01-81$ & 13,75 & 13,81 \\
\hline & $12-01-81$ & 13,50 & 13,56 \\
\hline \multirow{26}{*}{ después 12:30 pm } & $13-01-81$ & 13,30 & 13,36 \\
\hline & 14-01-81 (hasta 12:30 pm) & 13,20 & 13,26 \\
\hline & del 14-01-81 al 15-01-81 & 13,15 & 13,21 \\
\hline & 15-01-81 (desp. de 1 pm) & 12,94 & 13,00 \\
\hline & del 16-01-81 al 19-01-81 & 12,92 & 12,98 \\
\hline & $20-01-81$ & 12,82 & 12,88 \\
\hline & $21-01-81$ & 12,80 & 12,86 \\
\hline & $22-01-81$ & 12,75 & 12,81 \\
\hline & $23-01-81$ & 12,73 & 12,79 \\
\hline & del $26-01-81$ al $27-01-81$ & 12,70 & 12,76 \\
\hline & del 28-01-81 al 29-01-81 & 12,65 & 12,71 \\
\hline & del $30-01-81$ al $02-02-81$ & 12,64 & 12,70 \\
\hline & del 03-02-81 al 12-02-81 & 12,60 & 12,66 \\
\hline & 12-02-81 (des. 12:15 pm) & 14,00 & 14,06 \\
\hline & del 13-02-81 al 17-02-81 & 13,57 & 13,63 \\
\hline & del 17-02-81 al 18-02-81 & 13,81 & 13,87 \\
\hline & del 18-02-81 al 19-02-81 & 13,87 & 13,93 \\
\hline & del 19-02-81 al 20-02-81 & 13,93 & 13,99 \\
\hline & del $20-02-81$ al $23-02-81$ & 14,50 & 14,56 \\
\hline & del 23-02-81 al 24-02-81 & 15,50 & 15,56 \\
\hline & $25-02-81$ & 16,05 & 16,11 \\
\hline & del $25-02-81$ al $26-02-81$ & 17,04 & 17,10 \\
\hline & del $26-02-81$ al $27-02-81$ & 17,00 & 17,06 \\
\hline & del $27-02-81$ al $04-03-81$ & 16,75 & 16,81 \\
\hline & $05-03-81$ & 16,70 & 16,76 \\
\hline & 05-03-81 & 16,89 & 16,95 \\
\hline \multirow[t]{10}{*}{ (A)-- } & 06-03-81 & 16,84 & 16,90 \\
\hline & del 09-03-81 al 17-03-81 & 15,00 & 15,06 \\
\hline & $17-03-81$ & 15,00 & 17,42 \\
\hline & $18-03-81$ & 15,00 & 17,50 \\
\hline & $20-03-81$ & 15,00 & 17,55 \\
\hline & $23-03-81$ & 15,00 & 17,52 \\
\hline & 24-03-81 & 15,00 & 17,38 \\
\hline & $25-03-81$ & 15,00 & 17,04 \\
\hline & $26-03-81$ & 15,00 & 16,91 \\
\hline & $27-03-81$ & 15,00 & 17,12 \\
\hline
\end{tabular}




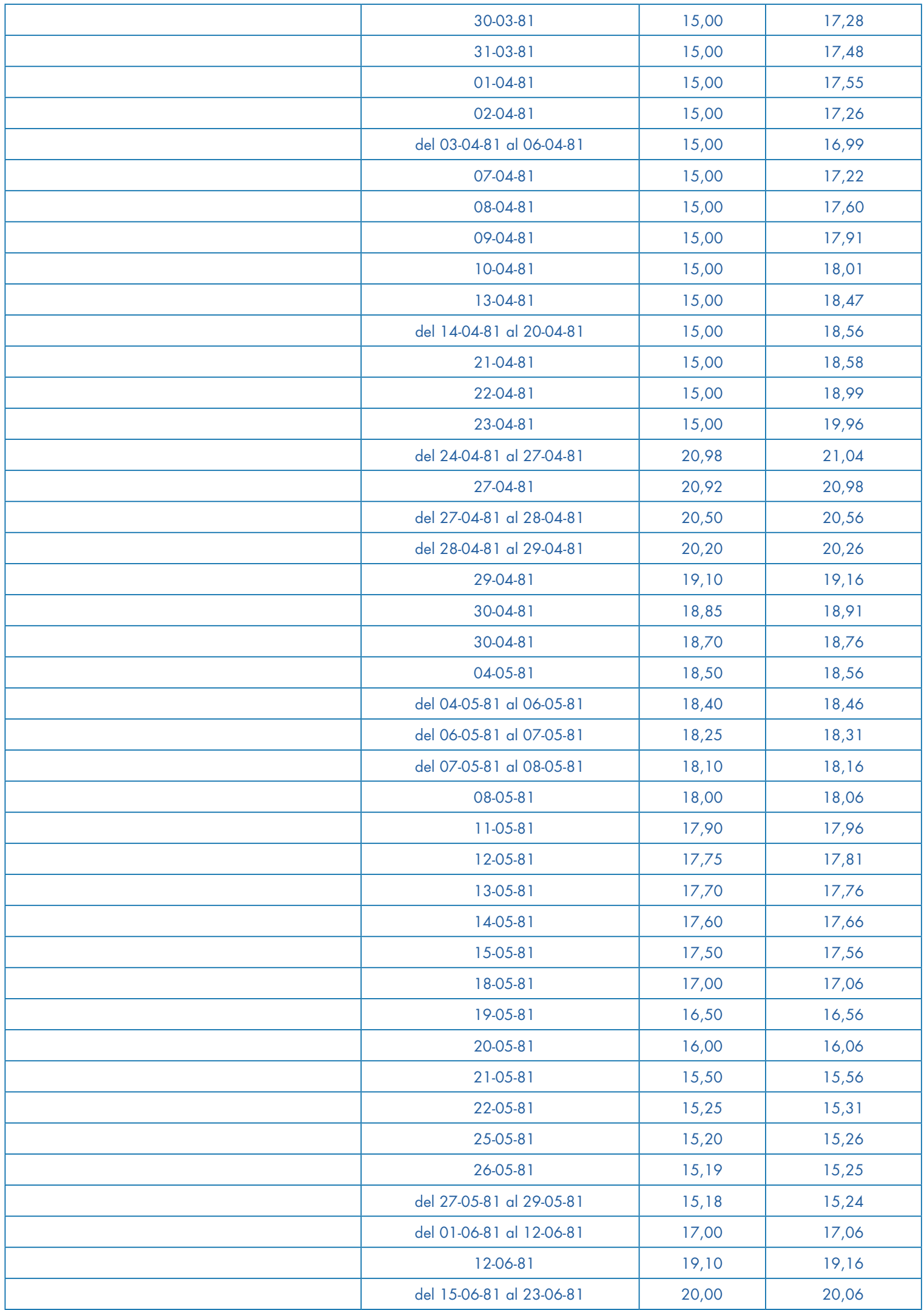




\begin{tabular}{|l|c|c|c|}
\hline (A)- & del 24-06-81 al 30-06-81 & 19,00 & 19,06 \\
\hline (B)- & del 01-07-81 al 07-07-81 & 18,94 & 19,00 \\
\hline & del 08-07-81 al 07-10-81 & 18,84 & 18,90 \\
\hline & del 08-10-81 al 15-10-81 & 30,00 & 30,06 \\
\hline & del 16-10-81 al 20-10-81 & 32,00 & 32,06 \\
\hline & del 23-10-81 al 02-1 1-81 & 38,00 & 38,18 \\
\hline & del 03-1 1-18 al 05-1 1-81 & 37,50 & 37,68 \\
\hline & del 06-1 1-81 al 10-11-81 & 36,80 & 36,98 \\
\hline & del 11-11-81 al 27-11-81 & 36,00 & 36,18 \\
\hline & del 30-11-81 al 17-12-81 & 35,80 & 35,98 \\
\hline & del 18-12-81 al 08-01-82 & 36,00 & 36,18 \\
\hline & del 11-01-82 al 14-01-82 & 35,70 & 35,88 \\
\hline & del 15-01-82 al 27-01-82 & 36,20 & 36,38 \\
\hline & del 28-01-82 al 19-02-82 & 37,00 & 37,18 \\
\hline (C)-- & del 22-02-82 al 18-03-82 & 37,20 & 37,38 \\
\hline & del 22-03-82 al 1 1-06-82 & 38,00 & 38,18 \\
\hline (D)- & del 12-06-82 al 28-08-82 & 38,00 & 38,25 \\
\hline (E)- & del 30-08-82 al 14-08-82 & 40,00 & 40,25 \\
\hline & del 15-09-82 al 01-12-82 & 40,00 & 40,30 \\
\hline & del 02-12-82 al 27-06-83 & 40,00 & 40,50 \\
\hline & del 28-06-83 al 22-09-83 & 41,00 & 41,50 \\
\hline & del 23-09-83 al 10-11-83 & 41,25 & 41,75 \\
\hline
\end{tabular}

Fuente: Banco de Costa Rica, documento facilitado por el BCCR. Para esta época, si bien se manejaba un tipo de cambio en el mercado, para los efectos oficiales se mantenía la venta de dólares a precio oficial. Se manejaba una doble realidad en esta materia. El precio del dólar en el mercado negro podía ser muy variado.

Para 1979 el tipo de cambio cierra en el mercado oficial en $\mathbb{\varangle} 8,60$, a diciembre de 1980 alcanza $\mathbb{\&} 14,31$ con un incremento del 66,40 \% en el tipo de cambio. En un solo año, de 1980 a diciembre de 1981, el dólar sube a $\llbracket 36,18$, lo que representó un aumento porcentual de $152 \%$. Este aumento de 219,23\% en el tipo de cambio generó en el poder adquisitivo una crisis sin precedentes en el país, situación que persiste hasta 1983, año en que el BCCR establece el mercado libre intercambiario, pero solo de referencia, lo cual para los primeros dos años logra controlar un poco la velocidad de la devaluación del colón. 
Tabla 3. Tipo de cambio nominal de Costa Rica.

Precio de compra, precio de venta y tasa de crecimiento en el periodo 1970-2020.

Promedio anual 1960-2020

\begin{tabular}{|c|c|c|c|c|}
\hline AÑO & $\begin{array}{l}\text { PRECIO } \\
\text { Compra }\end{array}$ & $\begin{array}{c}\text { PRECIO } \\
\text { Venta }\end{array}$ & $\begin{array}{l}\text { DIFERENCIA } \\
\text { PRECIO V-C }\end{array}$ & $\begin{array}{c}\text { VENTA } \\
\text { CAMBIO \% }\end{array}$ \\
\hline 1970 & 6,62 & 6,65 & 0,03 & \\
\hline 1971 & 8,57 & 8,60 & 0,03 & 29,32 \\
\hline 1972 & 8,57 & 8,60 & 0,03 & 0,00 \\
\hline 1973 & 8,54 & 8,60 & 0,06 & 0,00 \\
\hline 1974 & 8,54 & 8,60 & 0,06 & 0,00 \\
\hline 1975 & 8,54 & 8,60 & 0,06 & 0,00 \\
\hline 1976 & 8,54 & 8,60 & 0,06 & 0,00 \\
\hline 1977 & 8,54 & 8,60 & 0,06 & 0,00 \\
\hline 1978 & 8,54 & 8,60 & 0,06 & 0,00 \\
\hline 1979 & 8,54 & 8,60 & 0,06 & 0,00 \\
\hline 1980 & 14,25 & 14,31 & 0,06 & 66,40 \\
\hline 1981 & 36,00 & 36,18 & 0,18 & 152,83 \\
\hline 1982 & 40,00 & 40,50 & 0,50 & 11,94 \\
\hline 1983 & 43,15 & 43,65 & 0,50 & 7,78 \\
\hline 1984 & 47,50 & 47,50 & 0,00 & 8,82 \\
\hline 1985 & 53,45 & 53,95 & 0,50 & 13,58 \\
\hline 1986 & 58,50 & 59,25 & 0,75 & 9,82 \\
\hline 1987 & 68,75 & 69,75 & 1,00 & 17,72 \\
\hline 1988 & 79,00 & 80,00 & 1,00 & 14,70 \\
\hline 1989 & 83,85 & 84,85 & 1,00 & 6,06 \\
\hline 1990 & 102,55 & 104,55 & 2,00 & 23,22 \\
\hline 1991 & 134,10 & 136,75 & 2,65 & 30,80 \\
\hline 1992 & 136,74 & 138,07 & 1,33 & 0,97 \\
\hline 1993 & 150,61 & 152,17 & 1,56 & 10,21 \\
\hline 1994 & 164,51 & 165,63 & 1,12 & 8,85 \\
\hline 1995 & 194,43 & 195,37 & 0,94 & 17,96 \\
\hline 1996 & 219,91 & 220,31 & 0,40 & 12,77 \\
\hline 1997 & 244,04 & 244,53 & 0,49 & 10,99 \\
\hline 1998 & 271,19 & 271,65 & 0,46 & 11,09 \\
\hline 1999 & 297,96 & 298,41 & 0,45 & 9,85 \\
\hline 2000 & 317,74 & 318,30 & 0,56 & 6,67 \\
\hline 2001 & 341,40 & 341,94 & 0,54 & 7,43 \\
\hline 2002 & 378,39 & 379,05 & 0,66 & 10,85 \\
\hline 2003 & 418,04 & 419,01 & 0,97 & 10,54 \\
\hline 2004 & 457,58 & 459,64 & 2,06 & 9,70 \\
\hline 2005 & 495,65 & 497,71 & 2,06 & 8,28 \\
\hline 2006 & 515,84 & 519,95 & 4,11 & 4,47 \\
\hline
\end{tabular}




\begin{tabular}{|l|l|l|l|l|}
\hline 2007 & 495,23 & 500,97 & 5,74 & $-3,65$ \\
\hline 2008 & 550,08 & 560,65 & 10,57 & 11,91 \\
\hline 2009 & 558,67 & 571,81 & 13,14 & 1,99 \\
\hline 2010 & 507,85 & 518,09 & 10,24 & $-9,39$ \\
\hline 2011 & 505,35 & 518,33 & 12,98 & 0,05 \\
\hline 2012 & 502,07 & 514,32 & 12,25 & $-0,77$ \\
\hline 2013 & 495,01 & 507,80 & 12,79 & $-1,27$ \\
\hline 2014 & 533,31 & 545,53 & 12,22 & 7,43 \\
\hline 2015 & 531,94 & 544,87 & 12,93 & $-0,12$ \\
\hline 2016 & 548,18 & 561,10 & 12,92 & 2,98 \\
\hline 2017 & 566,42 & 572,56 & 6,14 & 2,04 \\
\hline 2018 & 604,39 & 611,75 & 7,36 & 6,84 \\
\hline 2019 & 567,06 & 573,36 & 6,30 & $-6,28$ \\
\hline 2020 & 610,53 & 617,30 & 6,77 & 7,66 \\
\hline
\end{tabular}

Fuente: Elaboración propia a partir de los datos del BCCR.

Tabla 4. Tipo de cambio mercado intercambiario de Costa Rica.

Precio de venta y promedio anual en el periodo 1983-2020

\begin{tabular}{|c|c|c|c|c|c|c|c|c|c|c|c|c|c|c|c|c|c|c|c|}
\hline & 2 ตes & 1204 & 1205 & 1 sas & $1 \mathrm{mo}$ & 1 sat & 100 & 1900 & $1 m$ & $1 m$ & $1 \mathrm{ws}$ & $1 \mathrm{~mol}$ & $1 \mathrm{~ms}$ & $18 \mathrm{~m}$ & $1 m$ & $1 \mathrm{mot}$ & $1 \mathrm{~mm}$ & 2000 & 2001 \\
\hline ENeteo & 4575 & 43.65 & 48.45 & sess & soss & $735 s$ & 30.15 & 85.45 & tosos & 138.65 & 193.12 & $18 \mathrm{~s}, 12$ & $167.6 s$ & 197.53 & 222.41 & 246.00 & 27406 & 290.35 & 3002 \\
\hline FIMIRO & 45.10 & 41,65 & 48,70 & 4,70 & 60.10 & 7420 & 0.55 & $\mathbf{n s , \infty}$ & 112.55 & 213,50 & $210 \mathrm{~s}$ & 351,62 & 362.95 & $1 n \pi$ & 22404 & 208.12 & 20,44 & 301,49 & 201.57 \\
\hline Meero & 44.75 & 43.65 & 49.25 & sass & 6050 & 7450 & $\operatorname{sens}$ & 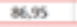 & 166.50 & 133.64 & $138 \% 5$ & 154.53 & $172 n$ & 201.92 & 236.07 & 250.32 & 27.08 & 300.35 & MBS.45 \\
\hline Aอง! & 465 & 41,65 & 49,50 & 5,25 & 61.25 & $7 n 05$ & seos & p.ss & 112.50 & 1325 & $230 \mathrm{~s}$ & 15,05 & 174,91 & zonת & 23060 & 252.5 & 202.50 & 301,32 & $34 \pi$ \\
\hline mavo & 4460 & 4.00 & 4985 & $5 s .85$ & 62.05 & $\sin$ & 81.55 & 8.95 & 1228 & 128.68 & 138.83 & 158.71 & 177.52 & 20500 & 250.87 & 254.44 & 283.93 & 306.63 & 30654 \\
\hline NNEO & 4160 & 4,25 & 50,6 & 56.45 & 6,5 & $\pi, 5$ & 3265 & n. $\mathbf{x}$ & 1245 & 24,28 & 801.14 & 156,00 & $172 n$ & 207,30 & 232.94 & 2565 & 20064 & 300,4 ? & nows \\
\hline WUO & 43.60 & 44.25 & $\$ 1.20$ & 56.65 & 6035 & 77.10 & 82.45 & 92.65 & 127.3 & 134.83 & 14281 & 15988 & 188.36 & 200000 & 23500 & 259.00 & 288.97 & 310,00 & 350.38 \\
\hline Acosto & 4160 & 4.25 & s1.,s & 57,05 & G10 & 77,5 & 2.00 & $\boldsymbol{N}, \boldsymbol{\infty}$ & $12 n$ & 23600 & 3466 : & 159,12 & ISAM & nus & 236.91 & 26169 & 20,73 & 311,24 & 328,36 \\
\hline Septereses & 4260 & 45.00 & $\$ 2,45$ & St.6s & 65.15 & 70.47 & 33.55 & *.00 & 132.5s & 136.56 & 14650 & 360.51 & 187.62 & nesst & 2647 & 264.07 & 232.62 & 313.35 & beass \\
\hline octuaser & 4260 & 40,00 & 52,25 & 57,55 & $0, \pi 5$ & $n \infty \infty$ & 206 & nos & 1213ss & 237,04 & $\operatorname{sen}, n$ & 360,27 & 190,16 & 316.31 & $200, \pi 7$ & 266.67 & $2 \mathrm{n}, 5 \mathrm{ss}$ & 315,01 & 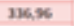 \\
\hline Noviemone & 4265 & 48.00 & $\$ 8.90$ & se.65 & 6.35 & ness & 34.5 & 101.80 & 135.15 & 137.53 & 150.35 & 364,09 & 198.06 & 21841 & 24200 & 298.17 & 236.42 & $336 n$ & 309.56 \\
\hline DHCEMARE & 0165 & 0,50 & s1,9s & 52,25 & 0.5 & $\infty, \infty$ & 405 & 10455 & $136, \pi$ & 213.07 & 152.17 & 365,63 & 193,37 & 20031 & 245.53 & 271.65 & $2 n, 4 !$ & 336,30 & H1,34 \\
\hline \multirow[t]{3}{*}{ PAOMETOO ANUUA" } & 43.97 & 4.9 & $\$ 1, \infty$ & 5653 & 62,60 & $\pi 0$ & $12 \times 4$ & 10,06 & 124 & 135, & 193,34 & 158.96 & 191.36 & 2005 & 231 & 2544 & 20.95 & 300,19: & 130.64 \\
\hline & & & & & & & & & & & & & & & & & & & \\
\hline & 2002 & 2003 & 2004 & 2005 & 2006 & 2000 & 2000 & 2000 & 2010 & 208 & 2012 & 2013 & 2014 & 2015 & 2ous & 2087 & 2018 & 2010 & 2000 \\
\hline ENtaO & 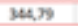 & $\$ 2.33$ & 42218 & 452.48 & 500.65 & sn.s & 496,34 & 565.16 & S65.11 & 58069 & 594.91 & $\operatorname{sen} \infty$ & 51963 & sases & $\operatorname{secs} 7$ & 561.42 & $5 n .27$ & 614.17 & 593.36 \\
\hline rtustino & 207,57 & ass.5s & 425,11 & 465,80 & 901,17 & 520,97 & 490,25 & 570.13 & scats & 507,61 & 526,20 & 506.4 & 551,54 & seasa & 502.20 & 564.16 & $\sin 2$ & $630, n$ & Sn1.54 \\
\hline mara & 350.39 & 38583 & 42250 & 46487 & 566.03 & 52065 & 499.69 & $\sec 15$ & 52878 & 506.16 & s313:5s & soe.65 & 58463 & 590.08 & $\sec 23$ & 567.34 & 565.31 & 600.36 & 587.39 \\
\hline Aองบ1 & 351,46 & $2 n 1,90$ & sinsa & 47207 & $\sec , 46$ & sso,cat & 497,21 & 37,97 & st6.04 & 506,44 & 500,50 & 500,46 & sse.16 & s) 11 & 5400 & 500,72 & $\operatorname{sen}, 3$ & $\sin , \infty$ & 571,46 \\
\hline Mavo & 356.74 & $305 / 4$ & 43473 & 475.19 & 511.30 & 520.76 & ș2ss & 581.63 & $\operatorname{ses} n \pi$ & StL.ts & 500.30 & 500.20 & ssass & 502.46 & $\sec 26$ & 590.12 & $\sec , n$ & $5 \times 0.54$ & S96.93 \\
\hline ग्रNO & 250,70 & nosso & S20101 & shea & 51406 & $530, n$ & $5 n, \pi$ & $\sin 91$ & 500.24 & 500,57 & 501.85 & 500,53 & $\sec c 6$ & 50097 & $\operatorname{sen} x 0$ & $\sin \pi$ & 500,04 & set, 4 & $\operatorname{sen}, 45$ \\
\hline wUo & 5660 & 402.22 & $442: 35$ & 441.S4 & 536.70 & seacs & 58664 & $\sin s e$ & $\sin 21$ & $520 \times 8$ & $\sec 28$ & 506,44 & Sesses & $\operatorname{sen} 80$ & 546.16 & 575.17 & 590.07 & 573.46 & $\sec 33$ \\
\hline Acosto & $x \in, 11$ & $\cos .55$ & 4400 & 464,74 & 529,34 & $520, \pi 5$ & 557,35 & 502,74 & ste.s & S:G,777 & 501,22 & 530,03 & 54,31 & 501.46 & stact & 57,42 & 575,67 & 575,36 & $5 \times 4,00$ \\
\hline SEPTEMARE & 96,12 & $\operatorname{coses}$ & $448 \times 2$ & 497.96 & 522.06 & 520,74 & 559.26 & sat.s & 512.94 & $5: 287$ & 500.31 & ser.57 & 545.52 & Sat. 04 & $5 S 400$ & 574.13 & 505.200 & $\operatorname{ses} 38 t$ & 606.68 \\
\hline octuaser & $3 n, s$ & 41254 & 5127 & $\$ x, 93$ & 529,63 & 531,36 & $\operatorname{sens1}$ & $\operatorname{sen} 31$ & 512.10 & 521,11 & 501,24 & 506,02 & $54 \pi$ & 50043 & 500.11 & 572.11 & 620,74 & sas,, 3 & 612,92 \\
\hline NOVEMEG & 35.88 & $415.5 \%$ & 455.58 & 430 & 538.78 & 500.25 & SBas & $\operatorname{ssin} 2 y$ & 51220 & 512.10 & 503.17 & ser.13 & 541.19 & ser & 584.40 & scess & 603.10 & 565.53 & 60873 \\
\hline DHCIMARE & $2 m, 06$ & 412,01 & ssact & $4 m, n$ & sas,ss & 300,97 & seoss & 57201 & steos & $\operatorname{sen}, 13$ & 514.12 & 500,50 & 545,53 & $\sec 0$ & 561.10 & 572.6 & 611,73 & 576,43 & 617,30 \\
\hline PAOMTOLO AYUA & 961.4 & 20058 & 40.16 & 400.02 & s112.4 & S11.42 & $\sin 91$ & smeat & 529.4 & s12.ss & $\sin n$ & $\operatorname{ses} n$ & Stat: & 50097 & 53206 & 572.0 & sniss & $\sin 40$ & 591..3 \\
\hline
\end{tabular}

Fuente: BCCR. 
Tabla 5. Tipo de cambio de Costa Rica. Precio de compra, precio de venta, aumentos de cada 50 colones y $\mathbf{n} .^{\circ}$ de días en ese valor

\begin{tabular}{|c|c|c|r|}
\hline Fecha & Compra & Venta & $\mathbf{N}^{\circ}$ días \\
\hline 6-jun-85 & 49,75 & 50,25 & 1981 \\
\hline 8-nov-90 & 98,40 & 100,15 & 1112 \\
\hline $24-$-nov-93 & 148,58 & 150,01 & 829 \\
\hline 2 -mar-96 & 199,27 & 200,06 & 754 \\
\hline 26 -mar-98 & 249,60 & 250,07 & 677 \\
\hline 1-feb-00 & 299,56 & 300,07 & 784 \\
\hline 26 -mar-02 & 349,54 & 350,11 & 472 \\
\hline 11 -jul-03 & 399,46 & 400,02 & 460 \\
\hline $13-$ oct-04 & 448,36 & 450,07 & 468 \\
\hline 24-ene-06 & 498,05 & 500,02 & 3800 \\
\hline 20-jun-16 & 537,14 & 549,74 & 858 \\
\hline 26-oct-18 & 594,80 & 599,40 & \\
\hline
\end{tabular}

Fuente: Elaboración propia a partir de los datos del BCCR.

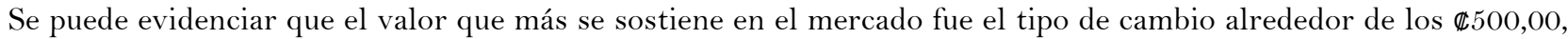
durante más de 10 años o 3.800 días, con una política económica de libertad cambiaria.

Tabla 6. Tipo de cambio de Costa Rica. Mayor diferencia entre el precio de compra y el precio de venta, 2014

\begin{tabular}{|c|c|c|r|}
\hline Fecha & Compra & Venta & Dif. V-C \\
\hline $17-m a r-14$ & 531,86 & 549,87 & 18,01 \\
\hline $14-m a r-14$ & 541,20 & 558,73 & 17,53 \\
\hline $18-m a r-14$ & 531,88 & 548,96 & 17,08 \\
\hline $19-m a r-14$ & 532,99 & 548,80 & 15,81 \\
\hline 20 -mar-14 & 533,05 & 548,77 & 15,72 \\
\hline 17 -abr-14 & 535,18 & 550,88 & 15,70 \\
\hline $18-a b r-14$ & 535,18 & 550,88 & 15,70 \\
\hline $19-a b r-14$ & 535,18 & 550,88 & 15,70 \\
\hline $21-a b r-14$ & 535,18 & 550,88 & 15,70 \\
\hline $20-a b r-14$ & 535,18 & 550,80 & 15,62 \\
\hline
\end{tabular}

Fuente. BNCR, cálculos propios.

En el año 2014 se presentan las mayores diferencias entre el tipo de cambio de compra y de venta. El 17 de marzo de 2014 fue el día donde se presentó la mayor diferencia histórica de $\mathbb{1} 18,01$. 


\section{CONCLUSIONES}

El tipo de cambio en los años sesenta se establece como un tipo de cambio fijo, producto de las negociaciones en Bretton Woods y los acuerdos con organismos como el FMI y la CEPAL, con la finalidad de mantener la competitividad en la región. En tanto el Gobierno no interfiriera directamente en la política cambiaria, a finales de los años setenta se contaba con un tipo de cambio oficial para trámite de transacciones, un tipo de cambio bancario y un tipo de cambio en el mercado negro.

A lo largo de estos sesenta años se pueden distinguir en el país ocho etapas en las que se pueden evidenciar políticas cambiarias definidas, dentro de las que se presentaron: desde el tipo de cambio fijo, las minidevaluaciones, sistema de bandas, un modelo de liberalización cambiaria, donde se vuelve fundamental la intervención del Gobierno mediante el manejo de las reservas monetarias internacionales para mantener estable el tipo de cambio, hasta llegar al sistema actual que corresponde a un sistema de flotación administrada.

A finales de los años setenta el país sufre la peor crisis cambiara de su historia, el tipo de cambio varía de $\mathbb{\$} 8,60$ en 1979 a $₫ 12,30$ en 1980. Para el 15 de agosto de 1982 el dólar alcanza el monto de $\mathbb{4} 40,30$, para alcanzar en enero de 1983 el valor de $\mathbb{4} 45,25$. De manera que, en un periodo de 3 años, se tiene un incremento del $426 \%$. Además, se cierra la década del ochenta con un tipo de cambio de $₫ 104,55$, para un incremento en una década de un $1115 \%$, mientras que en la década del 2006 al 2016, el incremento tendió de $0 \%$ a un $10 \%$ como máximo. Este es el periodo en el que Costa Rica presenta por más tiempo la estabilidad del tipo de cambio, con un valor alrededor de los $₫ 500,00$, lo cual representa que en el periodo de política de bandas cambiarias no se alcanza una diferencia de más menos $10 \%$.

Es a partir de 2014, a finales de la política de bandas cambiarias, que se presentan las mayores diferencias entre el precio de compra y venta del dólar en Costa Rica. El 17 de marzo de 2014 fue cuando se presentó la mayor diferencia histórica de $\mathbb{1} 18$,01.

En el 2015 con el establecimiento oficial del sistema de flotación administrada, si bien el tipo de cambio se ajusta por el libre juego de oferta y demanda, la intervención del Gobierno respaldado en las reservas monetarias internacionales, hace que el 7 de noviembre de 2018 en Costa Rica se alcance el monto más alto en el tipo de cambio del dólar, con un valor de $\mathbb{6} 631,30$. Razón por la cual debe intervenir el BCCR, con lo que en 10 días, se logra controlar el tipo de cambio a $\llbracket 604,10$ para el 17 de noviembre de 2018.

La pandemia del COVID-19, al afectar la economía mundial, permite que se mantenga el balance entre la oferta y la demanda del tipo de cambio. Si bien el sector turístico llegó a generar pocos ingresos en el 2020, otros sectores mantuvieron la producción, lo cual permitió que el tipo de cambio se mantuviese alrededor de los $\mathbb{6} 600,00$ durante el 2020 . 


\section{REFERENCIAS}

Blokhin, K. (2021). Fondo Monetario Internacional. eFXto. s.f. Consultado el 23 de febrero de 2021 . https://efxto.com/ diccionario/fondo-monetario-internacional-fmi

Chinchilla, D. (16 de marzo de 2014). El dólar: Una historia de amores y odios. La Nación. https://www.nacion.com/ revista-dominical/el-dolar-una-historia-de-amores-y-odios/N3HWPDUCYBHENPARS7DMIMFPQ4/story/

Delfino.CR. (1 de noviembre de 2018). El tipo de cambio al alza y las caras largas... también. Delfino. https://delfino. $\underline{\mathrm{cr} / 2018 / 11 / \text { el-tipo-de-cambio-al-alza-y }}$

Delgado-Montoya, E. (30 de enero de 2015). Banco Central eliminó la banda cambiaria y adopta flotación administrada del dólar. El Financiero. https://www.elfinancierocr.com/finanzas/banco-central-elimino-la-banda-cambiaria-yadopta-flotacion-administrada-del-dolar/RAMTCOAJ6VBHXLERBSH2EFKUKI/story/

Heilbroner, R. L. y Lester, C. T. (1987). Economía (7.a ed.). Prentice-Hall Hispanoamericana.

Krugman, P. R. y Obstfeld, M. (2006). Economía internacional. Teoría y política (7.a ed.). Pearson Educación.

Leitón, P. y Ramírez, E. (23 de agosto de 2018). Rodrigo Cubero, presidente del BCCR: 'La flexibilidad del tipo de cambio debe aumentar gradualmente'. La Nación. https://www.nacion.com/economia/politica-economica/ rodrigo-cubero-presidente-del-bccr-la/NP27ITEQ2ZBX3FLWV3X7IEPVNE/story/

Lizano, E. y López, G. (2006). Estudio Anual 2006. Régimen cambiario en Costa Rica. Academia de Centroamérica.

Mankiw, N. G. (2009). Principios de economía (5.a ed.). McGraw-Hill.

Parkin, M. (1995). Macroeconomía. Addison-Wesley Iberoamericana.

Saborío-Muñoz, G. (1997). Los regímenes cambiarios en Costa Rica: Evolución histórica y evaluación de algunas alternativas teóricas. Economía y Sociedad, 1(4), 99-114.

Salvatore, D. (1994). Economía internacional (3.a ed.). McGraw-Hill Interamericana.

Samuelson, P. A. y Nordhaus, D. W. (1992). Economía. Decimoctava.

Schatan, J. (1998). El saqueo de América Latina: deuda externa, neoliberalismo, globalización. Universidad Arcis.

Tacsan-Chen, R. (2001). Comercio Internacional. Editorial UNED.

Villasuso, J. M. (2000). Reformas estructurales y politica económica en Costa Rica. NU. CEPAL.

Wonnacott, P. y Wonnacott, R. (1981). Economía. McGraw-Hill. 